\title{
Role for Bim in termination of immune responses
}

The BH3-only pro-apoptotic BCL-2family member Bim is required for the death of activated T cells during the resolution of antiviral $\mathrm{T}$-cell responses in vivo, according to the latest findings from an Australian group now published in the Proceedings of the National Academy of Sciences.

Viral infection leads to rapid antigen-driven clonal expansion of $T$ cells that acquire effector functions and eliminate infected cells. Clearance of antigen is associated with a downregulation of the immune response and apoptosis of most antigenspecific T cells. Apoptosis can be mediated by signalling downstream of death receptors, members of the tumor-necrosis factor receptor (TNFR) family such as FAS (CD95) or TNFR1, or by pathways involving pro- and anti-apoptotic members of the BCL-2 family. Here, Pellegrini and colleagues investigated the involvement of Bim and CD95 in T-cell responses to infection with herpes simplex virus (HSV).

The kinetics of $\mathrm{CD}^{+}$T-cell responses to $\mathrm{HSV}$ in the lymph nodes of wild-type, $\mathrm{Bim}^{-/-}$and CD95-deficient lpr mice were similar, with infection leading to a rapid increase in the number of HSVspecific $\mathrm{CD}^{+} \mathrm{T}$ cells, which peaked at 7 days after infection. By 14 days, most of these $\mathrm{T}$ cells had disappeared from the lymph nodes. However, when the authors looked in the spleens of these mice, they saw an accumulation of HSV-specific $\mathrm{CD}^{+} \mathrm{T}$ cells in the Bimdeficient mice that did not occur in the wild-type or $l p r$ mice.

Why does loss of Bim function lead to increased numbers of virusspecific $\mathrm{T}$ cells in the spleens of $\mathrm{Bim}^{-/-}$mice? Further experiments showed that this accumulation did not result from prolonged activation or excessive proliferation of HSVspecific $\mathrm{CD} 8^{+} \mathrm{T}$ cells in these mice, or from differences in the trafficking of T cells. Neither was persistent viral infection responsible, as the kinetics of viral clearance were similar in $\mathrm{Bim}^{-/}$mice, lpr mice and wildtype mice. HSV-specific $\mathrm{CD} 8^{+} \mathrm{T}$ cells were shown to build up in the spleens of $\mathrm{Bim}^{-/-}$mice, but not in wild-type or lpr mice, due to these cells being resistant to apoptosis induced by cytokine withdrawal.

The authors conclude that neither CD95 nor Bim are required for viral clearance, but that Bim (and not CD95) is required for the apoptosis of virus-specific CD8 ${ }^{+} \mathrm{T}$ cells after clearance of HSV infection. Previous studies have shown that Bim is essential for the deletion of autoreactive $\mathrm{B}$ and $\mathrm{T}$ cells. Collectively, these results indicate that Bim is a crucial inducer of programmed cell death in the lymphoid lineage.

Jenny Buckland

\section{(19) References and links} ORIGINAL RESEARCh PAPER Pellegrini, M. et al. Shutdown of an acute $\mathrm{T}$ cell immune response to viral infection is mediated by the proapoptotic $\mathrm{Bcl}-2$ homology 3-only protein Bim. Proc. Natl Acad. Sci. USA 100, 14175-14180 (2003) FURTHER READING Bouillet, $\mathrm{P}$ et al. BH3-only Bcl-2 family member Bim is required for apoptosis of autoreactive thymocytes. Nature 415, 922-926 (2002)

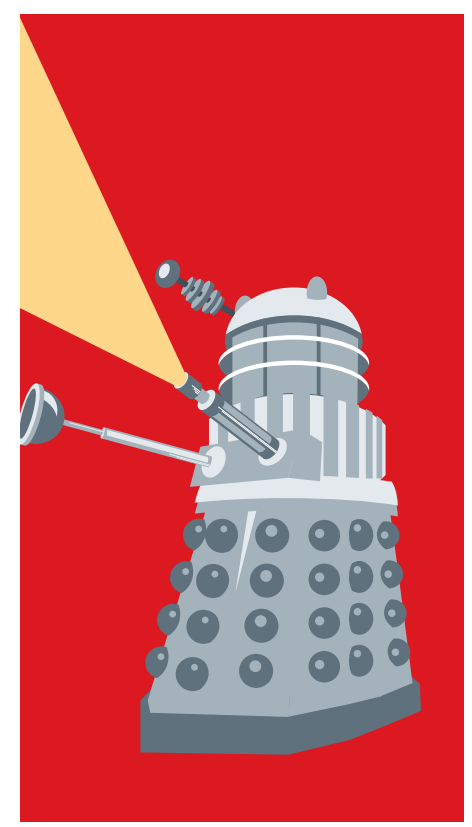

\section{IN BRIEF}

\section{T-CELL DEVELOPMENT}

\section{Critical roles for transcription factor GATA-3 in} thymocyte development.

Pai, S. -Y. Immunity 19, 863-875 (2003)

The zinc finger transcription factor GATA3 is expressed throughout thymocyte development, but its role in thymocyte differentiation is unknown. In this study, the authors generated mice in which GATA3 was deleted in the T-cell lineage at early and late stages of development. Absence of GATA3 expression in double-negative (DN) thymocytes resulted in reduced numbers of mature T cells and a partial arrest in development at the DN3 to DN4 transition, indicating a role for GATA3 at the $\beta$-selection checkpoint. Deletion of GATA3 later, after $\beta$-selection, showed that this transcription factor is also required at later stages of development in the generation of single-positive $\mathrm{CD} 4^{+}$thymocytes.

\section{TUMOUR IMMUNOLOGY}

Downregulation of HLA-A and Bw6, but not Bw4, allospecificities in leukemic cells. An escape mechanism from CTL and NK attack?

Demanet, C. et al. Blood 4 December 2003 (doi: 10.1182/blood-2003-07-2500)

Defects in MHC class I expression occur in most solid tumours as one mechanism of escape from T-cell attack, and they are often correlated with clinical outcome. This study used allele-specific human monoclonal antibodies to look at MHC class I expression by leukaemic cells. Downregulation of HLA-B expression was detected in most of the samples, but was restricted to HLA-Bw6 alleles. As HLA-Bw6 alleles - in contrast to HLA-Bw4 alleles - are not thought to be involved in inhibiting attack by natural killer (NK) cells, the authors suggest that the selective downregulation of HLA-Bw6 could prevent attack by cytotoxic T cells while avoiding NK-cell killing. Therefore, T-cell-based immunotherapeutic strategies should be developed that are specific for HLA-Bw4 alleles.

\section{TRANSPLANTATION}

\section{Pro-inflammatory functions of vascular endothelial} growth factor in alloimmunity.

Reinders, M. E. J. et al. J. Clin. Invest. 112, 1655-1665 (2003)

In both experimental models and human transplantation, the expression of vascular endothelial growth factor (VEGF) is associated with allograft rejection, but the mechanism for this is unknown. VEGF is a potent angiogenic factor, but it is also associated with pro-inflammatory responses. These authors show that in a humanized SCID mouse model, the infiltration of human T cells into skin allografts is enhanced by VEGF-induced endothelial expression of monocyte chemoattractant protein 1 and interleukin-8. In synergy with interferon- $\gamma($ IFN- $\gamma)$, VEGF also enhanced endothelial expression of the T-cell chemoattractant IFN-inducible protein 10 (IP-10). In line with this, a VEGF-specific antibody inhibited T-cell infiltration and acute rejection of heart transplants in mice. Therefore, VEGF-induced leukocyte recruitment is an important component of graft rejection, and VEGF might be a therapeutic target after transplantation. 